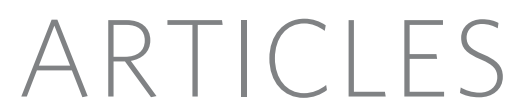

\title{
Hippocampal theta oscillations are travelling waves
}

\author{
Evgueniy V. Lubenov ${ }^{1}$ \& Athanassios G. Siapas ${ }^{1}$
}

\begin{abstract}
Theta oscillations clock hippocampal activity during awake behaviour and rapid eye movement (REM) sleep. These oscillations are prominent in the local field potential, and they also reflect the subthreshold membrane potential and strongly modulate the spiking of hippocampal neurons. The prevailing view is that theta oscillations are synchronized throughout the hippocampus, despite the lack of conclusive experimental evidence. In contrast, here we show that in freely behaving rats, theta oscillations in area CA1 are travelling waves that propagate roughly along the septotemporal axis of the hippocampus. Furthermore, we find that spiking in the CA1 pyramidal cell layer is modulated in a consistent travelling wave pattern. Our results demonstrate that theta oscillations pattern hippocampal activity not only in time, but also across anatomical space. The presence of travelling waves indicates that the instantaneous output of the hippocampus is topographically organized and represents a segment, rather than a point, of physical space.
\end{abstract}

Theta oscillations are a prominent 4-10-Hz rhythm in the hippocampal local field potential (LFP) of all mammals studied to date $e^{1-3}$, including humans ${ }^{4}$. During wakefulness they are associated with different speciesspecific behaviours, and they are invariably present during REM sleep ${ }^{2,3}$. In the rat, theta oscillations always accompany voluntary movement and active exploration ${ }^{2,5}$. Theta oscillations are essential for the normal functioning of the hippocampus, because manipulations that disrupt them produce behavioural impairments that mimic hippocampal lesions ${ }^{6,7}$. The importance of theta oscillations is underscored by the fact that they reflect subthreshold membrane potentials ${ }^{8-10}$ and strongly modulate the spiking, ${ }^{5,11,12,13}$ of hippocampal neurons. Furthermore, theta oscillations gate synaptic plasticity, because the timing of stimulation with respect to the phase of theta is important in determining the magnitude and direction of synaptic change ${ }^{14,15}$. Theta oscillations therefore offer macroscopic access to the internal clock of the hippocampal circuit, responsible for temporally patterning its operation. Such clocking is essential for the temporal coding of spatial information by place cells ${ }^{5,16}$, as evidenced by theta phase precession ${ }^{17-19}$. In addition to coding position, theta phase precession ensures that the order of place-cell firing over behavioural timescales (seconds) is preserved and compressed within individual theta cycles and inside the window of plasticity ${ }^{18}$. In the presence of spike-timing-dependent plasticity ${ }^{20}$, the resulting compression of temporal sequences offers a mechanism for the formation of hippocampal memory traces ${ }^{21}$. Furthermore, theta oscillations modulate activity not only in the hippocampus, but also in several subcortical, limbic and cortical structures ${ }^{22-24}$.

If theta oscillations can be thought of as a clock, what time is it in different parts of the hippocampus? In other words, how does the phase of theta oscillations depend on the anatomical location of the recorded LFP? It is well known that the amplitude and phase of theta oscillations depend on the depth, or more precisely the lamina, of the observed signal ${ }^{25-27}$, because several current dipoles located in different layers contribute to the macroscopic oscillation ${ }^{12,28-32}$. Conversely, it is believed that theta oscillations are robustly synchronized within each layer across the anatomical extent of the hippocampus ${ }^{28,32-34}$, despite some early evidence to the contrary ${ }^{26}$. The dominant view is therefore that theta oscillations are a global clock, and the anatomical extent of the hippocampus is synchronized with zero delay or, figuratively speaking, contained in a single time zone $\mathrm{e}^{32}$. In contrast to this view, we provide direct evidence that theta oscillations are not synchronized within stratum oriens of CA1, but are travelling waves that propagate predominantly along the septotemporal (long) hippocampal axis. Furthermore, we show that the firing of CA1 neurons is modulated in a wave pattern that is predicted by the LFP waves. Therefore, time in the hippocampus, as clocked by theta oscillations, is anatomically organized in a way similar to time on Earth-in a progression of local time zones.

\section{Depth profile of theta oscillations}

How can the depth dependence of theta phase be disentangled from systematic variations across the hippocampal surface? The solution requires recordings from a lamina in which the phase of theta oscillations shows little or no dependence on depth. We sought to determine whether such a lamina exists, and if so, whether it is sufficiently thick to allow reliable electrode positioning. To investigate this issue, we used multisite silicon probes with $50 \mu \mathrm{m}$ vertical site spacing (Supplementary Information, section 1.1), and quantified with high resolution the depth profile of theta oscillations in behaving (Fig. 1) and urethane-anesthetized rats (Supplementary Fig. 2). In agreement with several previous studies ${ }^{27,30,34}$, we found that theta oscillations start reversing their phase slightly below the pyramidal cell layer, as determined by the peak amplitude of sharp-wave-associated ripples and the reversal of sharp-wave polarity (Fig. 1e and Supplementary Fig. 4d). Moreover, there is a notable phase gradient throughout the dorsoventral (vertical) extent of stratum radiatum. In contrast, the phase of theta oscillations is practically constant throughout the vertical extent of a large slab of tissue (at least $400 \mu \mathrm{m}$ thick), just dorsal to the CA1 pyramidal cell layer and including the entire stratum oriens (Fig. 1b). Therefore, we can reliably study the spatial organization of theta oscillations by comparing LFPs recorded at any depth within stratum oriens of CA1, but at systematically different septotemporal and transverse coordinates in the hippocampus.

\section{Topography of theta phase offsets}

To sample theta oscillations topographically across the CA1 circuitry, we used microdrive arrays targeting rectangular grids spanning 

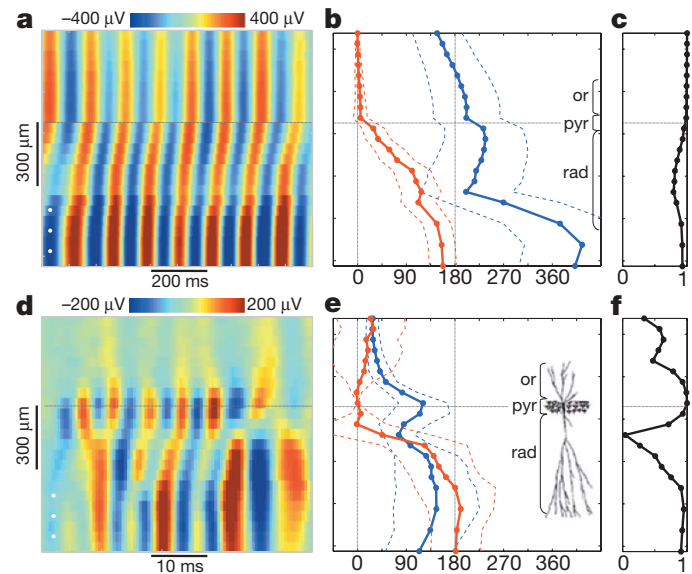

Figure 1 | Interlaminar depth profile of theta oscillations and ripples. a, Simultaneous CA1 recordings of LFPs filtered in the $4-10 \mathrm{~Hz}$ band during theta oscillations. In all panels data are ordered from most superficial (top) to deepest (bottom), with the pyramidal cell layer (pyr) marked by a horizontal line. White dots mark three inoperable sites where the image was interpolated. b, Theta amplitudes (blue, $\mu \mathrm{V}$ ) and phase deviations (red, deg) with respect to the top signal. Solid and dashed lines are the means and s.d., respectively. or, oriens; rad, radiatum. c, Theta coherence with respect to the top signal. $\mathbf{d}-\mathbf{f}$, Same as a-c but for LFPs filtered in the $80-250 \mathrm{~Hz}$ band during a ripple. Phase deviations and coherence are computed with respect to the signal at the pyramidal cell layer.

approximately $5 \mathrm{~mm}^{2}$ of the hippocampus (0.5-mm spacing) and allowing the independent depth adjustment of 24 tetrodes and four single reference electrodes (Supplementary Fig. 3). The arrays were chronically implanted and tetrodes were positioned in stratum oriens of CA1 on the basis of sharp-wave polarity and ripple power (Supplementary Information, section 1.2 and Supplementary Fig. 4). Figure 2 illustrates the anatomical position of one adjusted recording grid with respect to the hippocampus, and shows example recordings obtained while the rat ran on a linear track (Supplementary Figs 18-27).
Clear phase offsets were present in theta oscillations recorded in stratum oriens of CA1 from different locations on the grid (Fig. 2g), whereas their coherence across the grid was almost perfect (Supplementary Fig. 5 ). This is in contrast to the phase constancy of theta oscillations as a function of depth within stratum oriens discussed earlier (Fig. 1).

Next, we investigated whether the observed variability in the phase of theta oscillations is systematically organized across the hippocampus. To address this question (see Supplementary Information, section 1.3) we computed the instantaneous phase differences between theta oscillations recorded at each location on the grid and a reference tetrode in the centre of each grid (Fig. 3). These intralaminar phase differences exposed notable order and consistency across animals (Fig. 3). In particular, the relative phase of theta oscillations progressively advances from medial to lateral, whereas it is approximately constant along the rostrocaudal axis. Furthermore, the equiphase contours are roughly linear and are therefore well approximated by a planar (constant) phase gradient (analysis of variance (ANOVA) for multiple regression $P<10^{-9}, R^{2}>0.88$, in all three animals). The magnitude of the phase gradient in the three examples from different rats (Fig. 3a-c) was 21.4, 21.7 and $20.3^{\circ} \mathrm{mm}^{-1}$, and its anatomical orientation was $1^{\circ}, 16^{\circ}$ and $21^{\circ}$, respectively, with $0^{\circ}$ corresponding to the mediolateral and $-90^{\circ}$ to the rostrocaudal direction. The parsimonious explanation for the observed intralaminar theta phase profiles is that theta oscillations are in fact travelling plane waves that propagate with consistent speeds and directions across animals.

\section{Theta oscillations are travelling waves}

We analysed the instantaneous organization of theta oscillations and observed clear travelling plane waves (Fig. 4a, Supplementary Information, section 2 and Supplementary Movies 1-3). In fact, whenever robust theta oscillations were present in the hippocampus, they appeared as travelling waves, similar to the example shown in Fig. 4. The spatial wavelength and the direction of wave propagation were estimated at every point in time by fitting a plane to the instantaneous theta phase deviations (Supplementary Information, section 1.3 and Fig. 4b). This showed that theta waves can propagate not only in their

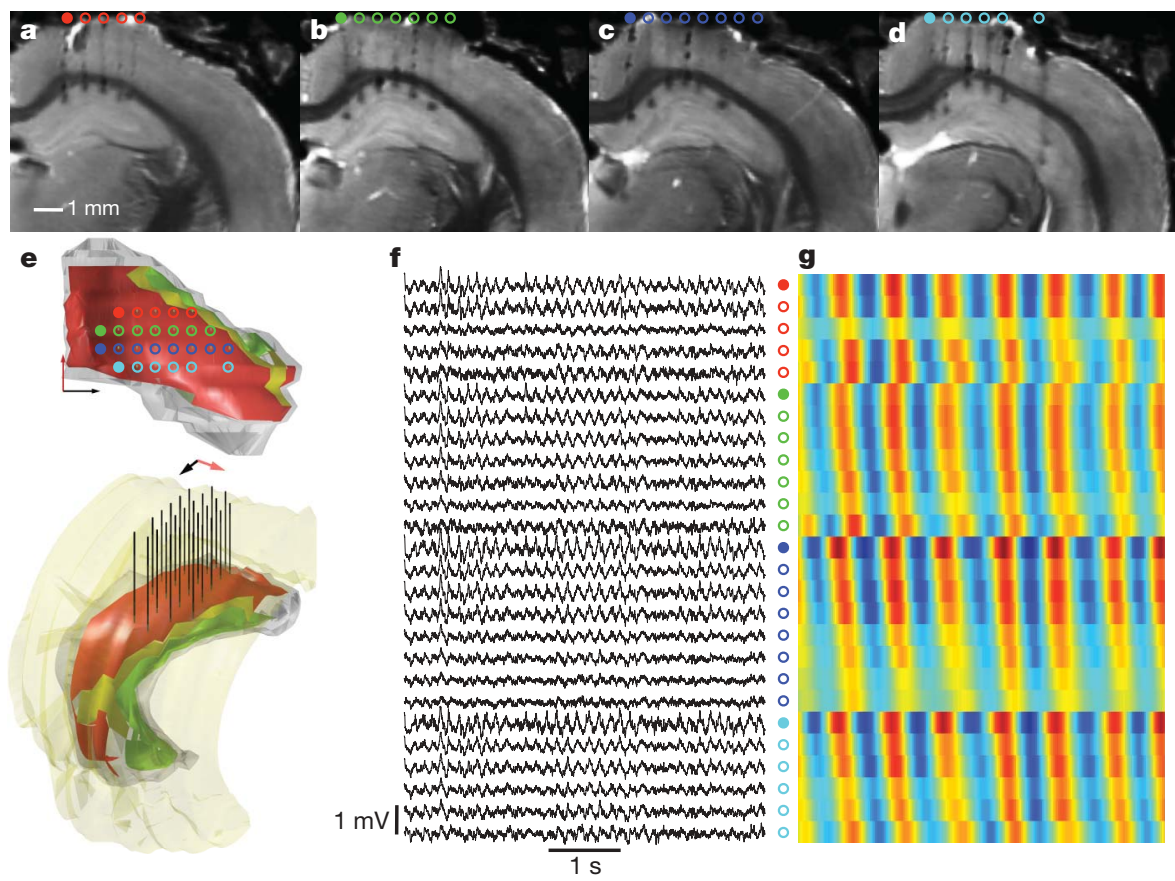

Figure 2 | Example of theta phase deviations. a-d, MRI coronal sections at bregma $-3.8(\mathrm{a}),-4.3(\mathrm{~b}),-4.8$ (c) and $-5.3(\mathrm{~d})(\mathrm{mm})$, showing final tetrode positions in the $4 \times 8$ grid. e, Bottom, three-dimensional model of the right hippocampus, with the CA1 pyramidal cell layer in red and tetrodes in black. The red arrow points rostrally and the black arrow points laterally. Top, top-down view with tetrodes marked by coloured circles. f, LFPs recorded from stratum oriens of CA1 using the grid in e during theta oscillations. Signals from each grid row are ordered from medial to lateral and stacked from rostral to caudal as indicated by the colour circles. $\mathbf{g}$, LFPs filtered in the $4-10 \mathrm{~Hz}$ band from the interval marked by the horizontal bar in $\mathbf{f}$. 
a
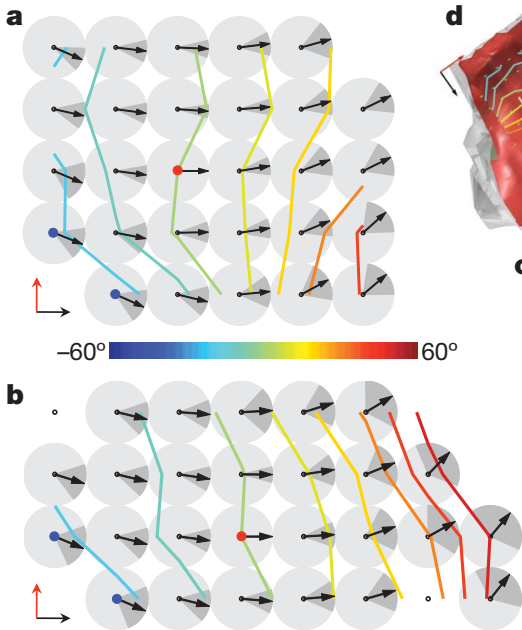

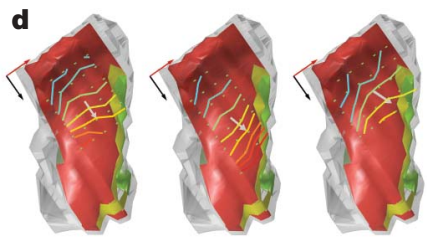

c

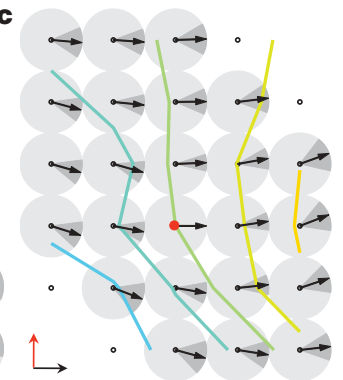

Figure 3 | Topographic intralaminar theta phase profiles. a-c, Grey circles mark grid points where LFPs were recorded over $45 \mathrm{~min}$ while three rats ran on a linear track. Black arrows show the means of the instantaneous theta phase deviations relative to a channel from the middle of each grid (red dots). Dark grey sectors indicate the corresponding s.d. The coloured lines

dominant direction but also within a range of $\pm 45^{\circ}$ around the dominant direction (Fig. 4c). The speed of theta wave propagation, $v$ (Fig. 4d), and the spatial wavelength, $\lambda$ (Fig. 4e), also showed a moderate level of variability (coefficient of variation $\sim 0.5$ ). The median values of the propagation direction (with respect to the mediolateral axis), speed, and spatial wavelength for each animal were as follows: $\theta=22^{\circ}, 3^{\circ}$ and $-17^{\circ}, v=87,80$ and $107 \mathrm{~mm} \mathrm{~s}^{-1}$, and $\lambda=13.6,11.1$ and $14.9 \mathrm{~mm}$. The mean propagation direction with respect to the septotemporal axis was $32^{\circ}$, with its transverse component pointing from distal to proximal CA1 (towards CA3). Wave parameter distributions were very similar across animals, although the within animal similarities were even higher, indicating the presence of small systematic individual differences (Fig. 4c-e).

\section{Travelling waves of spiking activity}

Because the LFP recorded at each point on the grid reflects the collective behaviour of the local neuronal population, the fact that theta oscillations are travelling waves indicates that neurons in CA1 must spike in a wave pattern consistent with the LFP wave (Supplementary are the contours of equal mean theta phase deviation. Grid points (blue dots) that may be in the subiculum have theta phase deviations consistent with CA1. $d$, The contours from a-c are replotted over a top-down view of the brain. Grey arrows show the mean direction and speed of wave propagation.

Information, section 1.4). We experimentally tested this prediction by positioning the tetrode grid within the CA1 pyramidal cell layer and recording the spiking activity of several neurons while rats ran on a linear track (Supplementary Figs 6, 8 and 9). To overcome the sparseness of firing on the timescale of a theta cycle (Supplementary Fig. 6b) we computed the mean phase of firing of spikes recorded at each point of the grid with respect to a single theta reference oscillation (Supplementary Figs 12 and 13). If spiking in CA1 is synchronized, the mean phase of firing at each point of the grid should be identical. In contrast, if spiking is organized as a travelling wave, the mean phase of firing should systematically advance across the grid in the direction of wave propagation. The latter is indeed the case when all recorded spikes are considered (Fig. 5, ANOVA for multiple regression, $5 \times 6$ grid: $P<0.007, R^{2}=0.41 ; 4 \times 8$ grid: $\left.P<0.0006, R^{2}=0.59\right)$, as well as when only the spikes of active pyramidal cells (Supplementary Figs 7-12) are analysed (Supplementary Fig. 14, ANOVA for multiple regression $P<0.03, R^{2}=0.51$, two-sample test of mean angles $P<0.001)$. Furthermore, the spiking wave parameters inferred from the topographic organization of mean phases match the characteristics

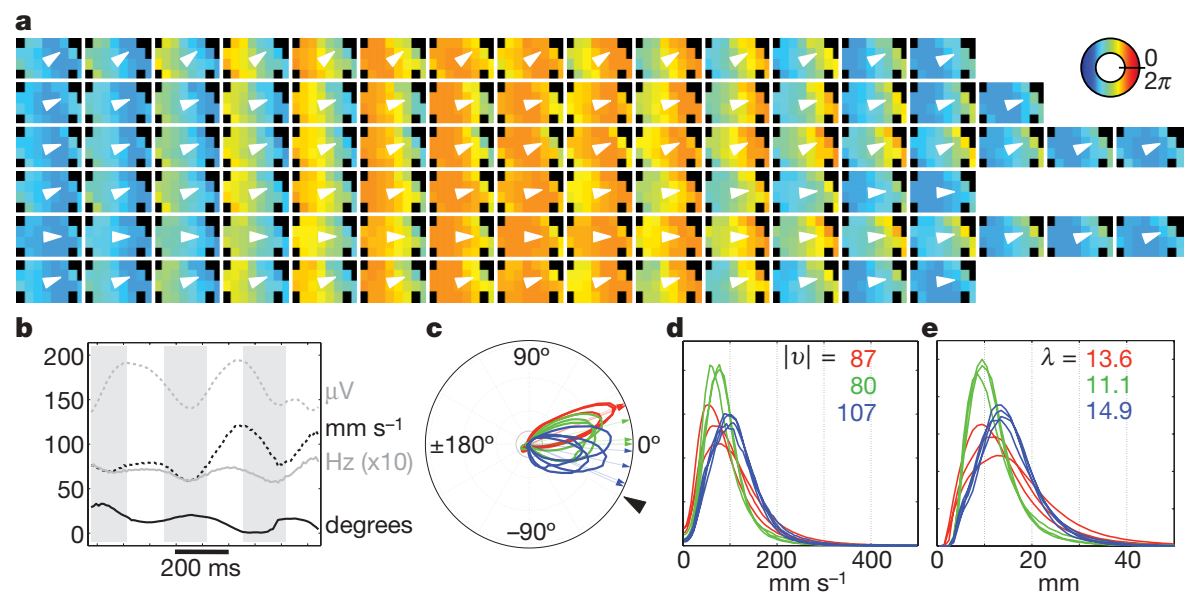

Figure 4 | Travelling wave example and wave parameter distributions. a, Frames show consecutive snapshots of theta oscillations across the recording grid, and white arrowheads mark the direction of wave propagation. Colours indicate the phase of theta at each grid location according to the circular colour map on the right. $\mathbf{b}$, Instantaneous wave parameters for the data in a: amplitude (dashed grey line), theta frequency (solid grey line), speed (dashed black line) and direction (solid black line) of wave propagation. The alternating white and grey stripes denote the six theta cycles shown as the six rows of frames in a. c-e, Parameter distributions from ten separate recording sessions: wave propagation direction $\left(0^{\circ}\right.$, mediolateral; black triangle, septotemporal) (c), wave propagation speed (d), and spatial wavelength (e). Different colours correspond to different animals, and the numbers show the median parameter values for each animal. 
a
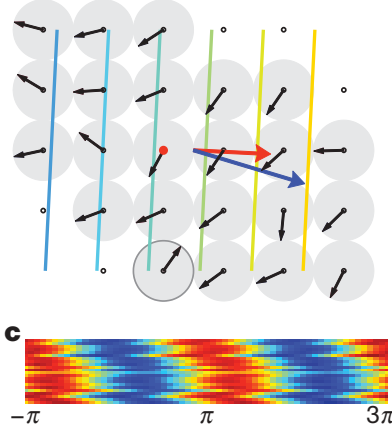
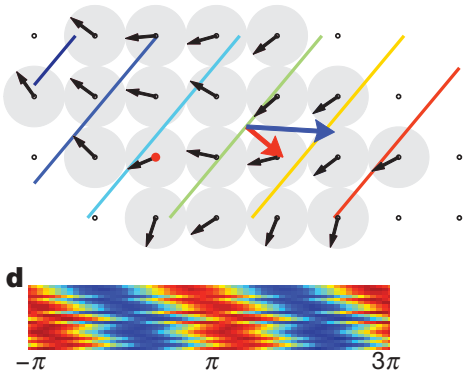

Figure 5 | Topographic profile of spiking theta phase preference. a, b, Multiunit mean phase of spiking at each grid location (black arrows) with respect to a single reference theta trace (red dot) in the $5 \times 6$ (a) and $4 \times 8$ (b) grids. The coloured lines are the level curves of the best linear fit to the unfolded mean phases. Large arrows mark the direction of wave propagation and spatial wavelength of spiking (red) and LFP (blue) waves. c, d, Each row shows the normalized multiunit theta phase distribution at a grid location and rows are ordered in the direction of spiking wave propagation. The slanted appearance demonstrates that the spiking probability is modulated in a wave pattern. Synchronous spiking would have resulted in vertical stripes.

of the theta wave seen in the LFPs (Fig. 5a, b). Parameter values were as follows $\left(5 \times 6\right.$ grid) LFP wave: $\theta=-17^{\circ}, \lambda=23.2 \mathrm{~mm}$, ANOVA $P<10^{-5}, R^{2}=0.62$; spiking wave: $\theta=-3^{\circ}, \lambda=15.4 \mathrm{~mm}$, ANOVA after marked outlier exclusion $P<0.007, R^{2}=0.41$; ( $4 \times 8$ grid) LFP wave: $\theta=-3^{\circ}, \lambda=17.4 \mathrm{~mm}$, ANOVA $P<0.0004, R^{2}=0.57$; spiking wave: $\theta=-40^{\circ}, \lambda=9.3 \mathrm{~mm}$, ANOVA $P<0.0006, R^{2}=0.59$. Notice that the LFP and spiking-wave parameters are extracted from nonoverlapping bands of the recorded signals and therefore represent independent measurements.

\section{Functional implications}

We have demonstrated that within the septal third of the hippocampus theta oscillations and CA1 firing are organized as travelling waves, propagating in a direction with a dominant septotemporal component, and wavelength commensurate with the long hippocampal axis. We do not know yet if the waves travel throughout the entire septotemporal axis of the hippocampus. Assuming, for now, that they do (Supplementary Fig. 15), we examine their potential functional implications. The anatomical projections of CA1 are systematically organized along the septotemporal axis with pronounced differences between the two poles ${ }^{35}$. For example, the septal third of CA1 projects to retrosplenial and perirhinal cortex, whereas projections to the hypothalamus, lateral septum, amygdala and medial prefrontal cortex preferentially originate from the temporal third of CA1 (refs 35-37). Travelling waves ensure that these distinct hippocampal targets receive peak CA1 input in a particular order. This may be important for the integration of heterogeneous information in downstream networks. Furthermore, there is growing evidence that the size of hippocampal place fields systematically increases along the septotemporal axis ${ }^{38-40}$. In the presence of travelling waves, this indicates that CA1 output may cycle from finer to coarser spatial resolution within each theta period.

Although the peak of CA1 activity travels septotemporally, an individual hippocampal neuron can still fire throughout the theta cycle, albeit with modulated intensity. Therefore, at each instant there are neurons discharging at every septotemporal level. A question remains as to how travelling waves inform what is represented by these populations of co-active cells. In addition to place specificity of firing ${ }^{16}$ (Supplementary Fig. 11), pyramidal neurons in the rat hippocampus show a strong correlation between place and phase of firing with respect to the local theta cycle, that is, theta phase precession ${ }^{17,19}$ (Supplementary Fig. 10). The phase precession relation allows one to infer the location of the place-field centres of the neurons that fire

in the course of a theta cycle (Fig. 6a). Consider, for example, a population of neurons with similar field sizes and four successive time windows in a theta cycle (Fig. 6a). Place specificity dictates that only the neurons with fields that overlap the rat's current location will fire (Fig. 6b), but phase precession further suggests that they will fire in sequence, rather than at once (Fig. 6c). Neurons with field centres behind the rat's location fire early in the theta cycle, whereas neurons with field centres ahead of the rat fire late (Fig. 6c). In this manner, a segment of space the length of a place field is traced in the course of a cycle. This effect, known as "compression of temporal sequences"18, has been established experimentally ${ }^{18,41}$ and presumably occurs at every septotemporal level. If theta oscillations are zero-lag synchronized, the compression of temporal sequences occurs in phase along the septotemporal axis and the instantaneous output of CA1 encodes a point of physical space (Fig. 6c). When the septotemporal expansion of place fields is considered ${ }^{38-40}$ and the nonlinearity of phase precession is accounted for ${ }^{17,18}$, the range of physical space represented by the hippocampus under synchronous oscillations depends on the phase of theta, shrinks to a point at least once, and is not consistently mapped on the septotemporal axis (Supplementary Figs 16 and 17a, c, e, bottom panel). Travelling theta waves introduce consistent theta phase offsets between different septotemporal levels, and so the compression of temporal sequences is systematically staggered along the septotemporal axis (Fig. 6d). In the presence of waves, at every instant a segment of physical space of characteristic length is topographically mapped along the septotemporal axis (Fig. 6d and Supplementary Figs $16,17 \mathrm{~b}, \mathrm{~d}, \mathrm{f}$, bottom panel). Time circularly shifts the encoded segment along the wave propagation direction, making it wrap around as if the septal and temporal poles were connected (Fig. 6d). So the fact that theta oscillations are travelling waves suggests that the instantaneous output of the hippocampus represents a segment, rather than a point, of physical space.

\section{Possible mechanisms}

Theoretical analysis of travelling waves in the cortex has suggested at least three distinct mechanisms ${ }^{42}$, and experimental data indicate that all three may be involved in theta oscillations. The first and simplest mechanism is delayed excitation from a single oscillator. This mechanism may apply to the medial septum, which is considered to be the principal
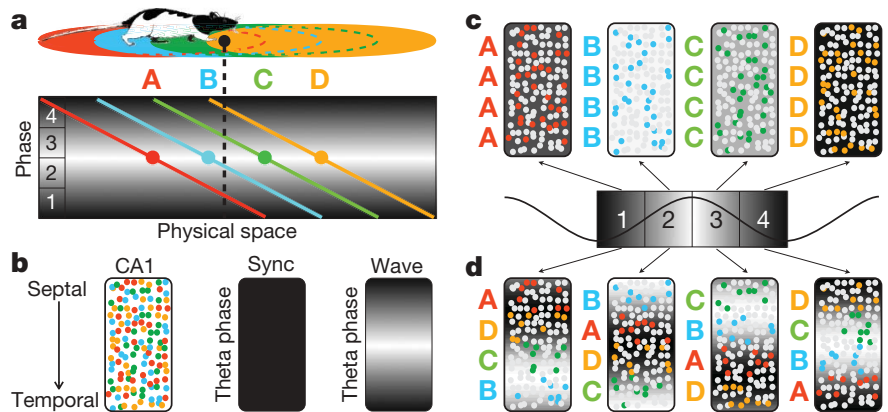

Figure 6 | Hippocampal representation of physical space. a, CA1 place fields with centres A-D overlapping the position of the rat (black line). The diagonal lines below illustrate the corresponding phase precession relations. The $x$ axis is physical location, and the $y$ axis is the local phase of theta oscillations (greyscale values). The numbered squares mark four time windows within the theta cycle. b, Unfolded map of CA1 with dots showing all place cells with fields overlapping the rat's position (colours correspond to field centres). The grey-scale panels show snapshots of the instantaneous theta phase throughout CA1 in the case of synchronized (sync) oscillations and travelling waves. c, Patterns of CA1 activity if theta oscillations are synchronized. Each panel is as in b, but only the cells active within each time window are coloured. At each time a single phase of theta is mapped across CA1, and consequently cells representing a single point in physical space are co-activated. d, If theta oscillations are travelling waves, at each time point a range of local theta phases is mapped across the hippocampus and consequently cells representing the segment $\mathrm{ABCD}$ are co-activated. 
pacemaker of theta oscillations and whose projections to the hippocampus are topographically organized along the septotemporal axis and travel through four separate routes with potentially different transmission delays ${ }^{35}$. The second mechanism is propagation of activity in an excitable network. This is relevant for the contribution of area CA3, because in disinhibited longitudinal slices pulses of activity propagate unattenuated throughout the length of CA3 $(10 \mathrm{~mm})$ with speeds of $0.1-0.15 \mathrm{~m} \mathrm{~s}^{-1}$ (refs 43, 44), matching the direction and speed of wave propagation we describe. The third mechanism requires a gradient of natural frequencies in a network of weakly coupled oscillators. Neurons in the entorhinal cortex have intrinsically oscillating membranes with natural frequencies in the theta range ${ }^{45,46}$. Furthermore, the natural frequencies are topographically organized and decrease in the dorsoventral direction ${ }^{47}$, therefore providing the conditions for intrinsic travelling waves within the entorhinal cortex. Similarly, neurons within CA1 itself exhibit theta frequency resonance ${ }^{48}$ and it has been argued that resonant frequencies decrease along the septotemporal axis ${ }^{39}$, consistent with the observed wave propagation direction. Finally, it is important to emphasize that the brain areas exhibiting theta oscillations or containing theta phase-locked neurons are not connected in a simple feedforward network, but are part of a feedback loop with elaborate interconnection topographies. Therefore, theta resonance at the level of individual neurons $s^{45-48}$, local interactions between neuronal populations ${ }^{49}$, and global coupling between areas through connections with various transmission delays ${ }^{50}$ all play a part in determining the spatiotemporal characteristics of theta oscillations throughout the loop. Given the above considerations, we predict that theta oscillations are travelling waves not only in CA1, but also in dentate gyrus, CA3, subiculum and the entorhinal cortex.

Travelling waves with consistent propagation parameters within a circuit represent topographic maps of temporal activation order. Therefore, in conjunction with other known topographies and local circuit properties, they can elucidate not only the global features of information representation but also the direction of information flow and the nature of information processing.

\section{METHODS SUMMARY}

Long-Evans male rats, 3-6 months old, were used in all experiments. Chronic recordings were obtained while rats ran on a $180-\mathrm{cm}$ linear track for water reward. Acute recordings were obtained under urethane $\left(1.3 \mathrm{~g} \mathrm{~kg}^{-1}\right)$ anaesthesia. Two skull screws above the contralateral cerebellum and cortex served as ground and reference in all recordings.

Multisite silicon probe experiments. Rats were implanted chronically $(n=2)$ and acutely $(n=2)$ with multisite silicon probes targeting bregma $=-3.9$, lateral $=2.7$ (NeuroNexus Technologies, probe 2b: 54 sites in two staggered columns $43 \mu \mathrm{m}$ apart, $50 \mu \mathrm{m}$ vertical site spacing). During surgery, probes were stereotactically lowered and secured so that the middle of their recording vertical extent was approximately at the CA1 pyramidal cell layer.

Two-stage chronic microdrive array experiments. Rats $(n=3)$ were implanted with custom arrays targeting rectangular grids $(6 \times 5,4 \times 8$ and $5 \times 6)$, allowing the independent depth adjustment of 24 tetrodes and four reference electrodes. In all grids, probe spacing was $0.5 \mathrm{~mm}$ and the target of the caudomedial grid corner was bregma $=-5.3$, lateral $=1.0$. In phase 1 , tetrodes were positioned in stratum oriens of CA1 and the data used in the LFP theta wave analysis were obtained. In phase 2, tetrodes were lowered to the CA1 pyramidal cell layer and the data used in the spiking wave analysis were recorded.

Electrode position reconstruction. Sharp-wave polarity analysis was used during phase 1 to confirm the depth of all tetrodes within stratum oriens above CA1 (Supplementary Information, section 1.2.2 and Supplementary Fig. 1a). At the conclusion of phase 2 , the final location of each tetrode tip was marked by an electrolytic lesion ( $10 \mu \mathrm{A}$ anodal current for $15 \mathrm{~s})$, brains were fixed, and a highresolution structural MRI scan was performed to reconstruct electrode tracks in three-dimensions (Supplementary Fig. 1c).

\section{Received 7 February; accepted 18 March 2009.}

Published online 17 May 2009.

1. Green, J. D. \& Arduini, A. A. Hippocampal electrical activity in arousal. J. Neurophysiol. 17, 533-557 (1954).

2. Vanderwolf, C. H. Hippocampal electrical activity and voluntary movement in the rat. Electroencephalogr. Clin. Neurophysiol. 26, 407-418 (1969).
3. Winson, J. Interspecies differences in the occurrence of theta. Behav. Biol. 7, 479-487 (1972).

4. Arnolds, D. E., Lopes da Silva, F. H., Aitink, J. W., Kamp, A. \& Boeijinga, P. The spectral properties of hippocampal EEG related to behaviour in man.

Electroencephalogr. Clin. Neurophysiol. 50, 324-328 (1980).

5. O'Keefe, J. \& Nadel, L. The Hippocampus as a Cognitive Map (Oxford Univ. Press, 1978).

6. Winson, J. Loss of hippocampal theta rhythm results in spatial memory deficit in the rat. Science 201, 160-163 (1978).

7. Mitchell, S. J., Rawlins, J. N., Steward, O. \& Olton, D. S. Medial septal area lesions disrupt theta rhythm and cholinergic staining in medial entorhinal cortex and produce impaired radial arm maze behavior in rats. J. Neurosci. 2, 292-302 (1982).

8. Fujita, Y. \& Sato, T. Intracellular records from hippocampal pyramidal cells in rabbit during theta rhythm activity. J. Neurophysiol. 27, 1012-1025 (1964).

9. Leung, L. S. \& Yim, C. Y. Intracellular records of theta rhythm in hippocampal CA1 cells of the rat. Brain Res. 367, 323-327 (1986).

10. Vertes, R. P. \& Kocsis, B. Brainstem-diencephalo-septohippocampal systems controlling the theta rhythm of the hippocampus. Neuroscience 81, 893-926 (1997).

11. Ranck, J. B. Jr. Studies on single neurons in dorsal hippocampal formation and septum in unrestrained rats. I. Behavioral correlates and firing repertoires. Exp. Neurol. 41, 461-531 (1973).

12. Buzsaki, G., Leung, L. W. S. \& Vanderwolf, C. H. Cellular bases of hippocampal EEG in the behaving rat. Brain Res. 287, 139-171 (1983).

13. Fox, S. E., Wolfson, S. \& Ranck, J. B. Jr. Hippocampal theta rhythm and the firing of neurons in walking and urethane anesthetized rats. Exp. Brain Res. 62, 495-508 (1986).

14. Pavlides, C., Greenstein, Y. J., Grudman, M. \& Winson, J. Long-term potentiation in the dentate gyrus is induced preferentially on the positive phase of thetarhythm. Brain Res. 439, 383-387 (1988)

15. Huerta, P. T. \& Lisman, J. E. Heightened synaptic plasticity of hippocampal CA1 neurons during a cholinergically induced rhythmic state. Nature 364, 723-725 (1993).

16. O'Keefe, J. \& Dostrovsky, J. The hippocampus as a spatial map. Preliminary evidence from unit activity in the freely-moving rat. Brain Res. 34, 171-175 (1971)

17. O'Keefe, J. \& Recce, M. L. Phase relationship between hippocampal place units and the EEG theta rhythm. Hippocampus 3, 317-330 (1993).

18. Skaggs, W. E., McNaughton, B. L., Wilson, M. A. \& Barnes, C. A. Theta phase precession in hippocampal neuronal populations and the compression of temporal sequences. Hippocampus 6, 149-172 (1996).

19. Huxter, J., Burgess, N. \& O'Keefe, J. Independent rate and temporal coding in hippocampal pyramidal cells. Nature 425, 828-832 (2003).

20. Debanne, D., Gahwiler, B. H. \& Thompson, S. M. Long-term synaptic plasticity between pairs of individual CA3 pyramidal cells in rat hippocampal slice cultures. J. Physiol. (Lond.) 507, 237-247 (1998).

21. Lisman, J. E. Relating hippocampal circuitry to function: recall of memory sequences by reciprocal dentate-CA3 interactions. Neuron 22, 233-242 (1999)

22. Pedemonte, M., Pena, J. L. \& Velluti, R. A. Firing of inferior colliculus auditory neurons is phase-locked to the hippocampus theta rhythm during paradoxical sleep and waking. Exp. Brain Res. 112, 41-46 (1996).

23. Alonso, A. \& Garcia-Austt, E. Neuronal sources of theta rhythm in the entorhinal cortex of the rat. II. Phase relations between unit discharges and theta field potentials. Exp. Brain Res. 67, 502-509 (1987).

24. Siapas, A. G., Lubenov, E. V. \& Wilson, M. A. Prefrontal phase locking to hippocampal theta oscillations. Neuron 46, 141-151 (2005).

25. Green, J. D., Maxwell, D. S., Schindler, W. J. \& Stumpf, C. Rabbit EEG 'theta' rhythm: its anatomical source and relation to activity in single neurons. J. Neurophysiol. 23, 403-420 (1960).

26. Petsche, H. \& Stumpf, C. Topographic and toposcopic study of origin and spread of the regular synchronized arousal pattern in the rabbit. Electroencephalogr. Clin. Neurophysiol. 12, 589-600 (1960).

27. Winson, J. Patterns of hippocampal theta rhythm in the freely moving rat. Electroencephalogr. Clin. Neurophysiol. 36, 291-301 (1974).

28. Bland, B. H., Anderson, P. \& Ganes, T. Two generators of hippocampal theta activity in rabbits. Brain Res. 94, 199-218 (1975).

29. Leung, L. W. Model of gradual phase shift of theta rhythm in the rat. J. Neurophysiol. 52, 1051-1065 (1984).

30. Buzsaki, G., Czopf, J., Kondakor, I. \& Kellenyi, L. Laminar distribution of hippocampal rhythmic slow activity (RSA) in the behaving rat: current-source density analysis, effects of urethane and atropine. Brain Res. 365, 125-137 (1986).

31. Kocsis, B., Bragin, A. \& Buzsaki, G. Interdependence of multiple theta generators in the hippocampus: a partial coherence analysis. J. Neurosci. 19, 6200-6212 (1999).

32. Buzsaki, G. Theta oscillations in the hippocampus. Neuron 33, 325-340 (2002).

33. Bullock, T. H., Buzsaki, G. \& McClune, M. C. Coherence of compound field potentials reveals discontinuities in the CA1-subiculum of the hippocampus in freely-moving rats. Neuroscience 38, 609-619 (1990).

34. Bragin, A. et al. Gamma $(40-100 \mathrm{~Hz})$ oscillation in the hippocampus of the behaving rat. J. Neurosci. 15, 47-60 (1995).

35. Amaral, D. G. \& Witter, M. P. in The Rat Nervous System (ed. Paxinos G.) Ch. 21, 443-493 (Academic, 2004). 
36. van Groen, T. \& Wyss, J. M. Extrinsic projections from area CA1 of the rat hippocampus: olfactory, cortical, subcortical, and bilateral hippocampal formation projections. J. Comp. Neurol. 302, 515-528 (1990).

37. Cenquizca, L. A. \& Swanson, L. W. Spatial organization of direct hippocampal field CA1 axonal projections to the rest of the cerebral cortex. Brain Res. Rev. 56, 1-26 (2007).

38. Jung, M. W., Wiener, S. I. \& McNaughton, B. L. Comparison of spatial firing characteristics of units in dorsal and ventral hippocampus of the rat. J. Neurosci. 14, 7347-7356 (1994).

39. Maurer, A. P., Vanrhoads, S. R., Sutherland, G. R., Lipa, P. \& McNaughton, B. L. Selfmotion and the origin of differential spatial scaling along the septo-temporal axis of the hippocampus. Hippocampus 15, 841-852 (2005).

40. Kjelstrup, K. B. et al. Finite scale of spatial representation in the hippocampus. Science 321, 140-143 (2008).

41. Huxter, J. R., Senior, T. J., Allen, K. \& Csicsvari, J. Theta phase-specific codes for two-dimensional position, trajectory and heading in the hippocampus. Nature Neurosci. 11, 587-594 (2008)

42. Ermentrout, G. B. \& Kleinfeld, D. Traveling electrical waves in cortex: insights from phase dynamics and speculation on a computational role. Neuron 29, 33-44 (2001).

43. Miles, R., Traub, R. D. \& Wong, R. K. Spread of synchronous firing in longitudinal slices from the CA3 region of the hippocampus. J. Neurophysiol. 60, 1481-1496 (1988).

44. Traub, R. D. \& Miles, R. Neuronal Networks of the Hippocampus Ch. 6, 119-156 (Cambridge Univ. Press, 1991)

45. Alonso, A. \& Llinas, R. R. Subthreshold $\mathrm{Na}^{+}$-dependent theta-like rhythmicity in stellate cells of entorhinal cortex layer II. Nature 342, 175-177 (1989).
46. Haas, J. S. \& White, J. A. Frequency selectivity of layer II stellate cells in the medial entorhinal cortex. J. Neurophysiol. 88, 2422-2429 (2002).

47. Giocomo, L. M., Zilli, E. A., Fransen, E. \& Hasselmo, M. E. Temporal frequency of subthreshold oscillations scales with entorhinal grid cell field spacing. Science 315 , 1719-1722 (2007).

48. Leung, L. S. \& Yu, H.-W. Theta-frequency resonance in hippocampal CA1 neurons in vitro demonstrated by sinusoidal current injection. J. Neurophysiol. 79, 1592-1596 (1998).

49. Cobb, S. R., Buhl, E. H., Halasy, K., Paulsen, O. \& Somogyi, P. Synchronization of neuronal activity in hippocampus by individual GABAergic interneurons. Nature 378, 75-78 (1995).

50. Miller, R. Cortico-Hippocampal Interplay and the Representation of Contexts in the Brain Ch. 9, 159-188 (Springer-Verlag, 1991).

Supplementary Information is linked to the online version of the paper at www.nature.com/nature.

Acknowledgements We thank C. Wierzynski, M. Gu, G. Laurent, E. Schuman and A. Tolias for critical discussions and comments on the manuscript. We also thank the Caltech Brain Imaging Center and D. Procissi for the MRI brain scans. This work was supported by the Caltech Information Science and Technology Center for Biological Circuit Design, a 21st Century McDonnell Foundation Award, the Bren Foundation, and the McKnight Foundation.

Author Information Reprints and permissions information is available at www.nature.com/reprints. Correspondence and requests for materials should be addressed to A.G.S. (thanos@caltech.edu) or E.V.L. (lubenov@caltech.edu). 